tion we present, they are now more critical of the information they get from other sources and that includes pharmaceutical reps."

The Dalhousie program, which began in 200I, has annually received between \$200 o00 and \$300 ooo to conduct I or 2 reviews per year on topics chosen through a provincial survey of doctors.

Dalhousie disseminates its findings through office visits with physicians but its 4 counterparts in other provinces employ a range of methods.

Saskatchewan's RxFiles Academic Detailing Program visits roughly two-thirds of the province's physicians annually, but

\title{
"This is the kind of information we need"
}

$\mathrm{T}$ here's no doubt in the mind of clinical pharmacist Dr. Aaron Tejani, the co-ordinator of clinical research and drug information for British Columbia's Fraser Health Authority (FHA), that academic detailing has a indispensable role to play in the daily decisions that physicians and pharmacists make.

Tejani, whose duties include serving as pointman for the FHA's I2 acute care hospitals in meetings with pharmaceutical reps, says that becomes apparent many days that he meets pharmaceutical detailers. Last summer, for example, Tejani met with a Pfizer Inc. representative who pitched the use of acetylcholinesterase inhibitor donepezil (sold as Aricept for the treatment of Alzheimer's disease) to treat mild cognitive impairment (MCI), an off-label indication.

Tejani promptly hauled out a Therapeutic Letter, an academic detailing publication produced by the University of British Columbia's Department of Pharmacology and Therapeutics (April-August 2005;56). It indicated: that an unpublished clinical trial of the drug involving patients with MCI demonstrated no benefit in terms of preventing or delaying progression toward Alzheimer's disease; that several trials had suggested just $\mathrm{r} 6 \%$ of people with MCI go on to develop Alzheimer's; and that there wasn't a known correlation between the 2 disease states.

"A I6\% conversion rate is relatively small, so treating all people with MCI would be, in my mind, dangerous, especially when you have trials showing some of these drugs cause harm," Tejani says.

Pfizer spokesperson Christine Antoniou says the promotion of off-label use of drugs "is not standard practice and our representatives receive a lot of training and regular reminders that off-label promotion is not at all something that we want to do. We hope it's an isolated incident and we're looking into it."

Tejani says off-label promotion is common and described a number of similar pitches, including ones for off-label use of Recombinant Factor 7 for stroke patients, and moxifloxacin eyedrops for people undergoing cataract surgery. Pharmacy representatives have even urged FHA staff to lobby regulators to approve a drug for off-label indications, Tejani adds.

In 2005 , the FHA invited drug company representatives to a forum, where expectations regarding the accuracy and quality of clinical evidence presented by drug detailers were spelled out. "We told them: 'This is what we feel is appropriate, and this is where we think you will be crossing the line."

Tejani says such incidents demonstrate the value of optimal prescribing initiatives like therapeutics letters or academic detailing visits. "It [does away] with clinical opinion and bias. Instead, it simply states what we know and what we don't know about the evidence... [and] this is the kind of fact-based information clinicians and patients need to make informed decisions." - Wayne Kondro, CMAJ

Program Director Loren Regier says the Io-year-old, \$315 ooo per year initiative also conducts sessions at medical conferences, issues newsletters and email updates through the Saskatchewan Medical Association, and electronically compiles a compendium of its drug comparison charts, which it publishes periodically as a book that is distributed to every doctor in the province.

The next edition, slated for release this month, will cover over 55 therapeutic areas. "We're getting to be pretty comprehensive on the core areas."

The discontinued 7-year-old Alberta Drug Utilization Program (ADUP), by contrast, relied primarily on written reports to disseminate findings. Two years ago it began meeting with physicians face-to-face, says Program Administrator Don Phillipon, a professor of strategic management and health policy at the University of Alberta. The $\$ 500$ ooo per year program provided visits to physicians in 2 (Calgary and David Thompson) of Alberta's 9 health regions, reaching about Ioo physicians. When it was axed, Calgary authorities were so disappointed they found a way to resuscitate it within city boundaries.

Phillipon can't comprehend why ADUP was scuttled. "It's hilarious.... I teach a course comparing health systems, and pharmacy is one of the areas we comment on because this is the area of the Canadian health system that's most disorganized when you compare it to other countries with universal health systems. And here was an initiative that was really looking at utilization issues and it was discontinued."

Alberta Health and Wellness Communications Director Michael Shields says ADUP's limited scale proved its undoing. "The program, while providing evidence of effectiveness in changing prescriber behaviour, was not viewed as cost-effective if scaled to the entire prescriber community. There are over 7100 prescribers registered in $\mathrm{Al}$ berta, ADUP reached only 250 physicians in 2 areas in the province."

Prescription Information Services in Manitoba faces a similar fate. Executive Director Shawn Bugden says future funding for the $\$ 300$ o0o, 4-year initiative is in abeyance, despite its self-evident value. "It means that a physician can sit down 